\title{
Dictynna
}

Dictynna

Revue de poétique latine

$9 \mid 2012$

Varia

\section{Horace et la question de l'imitatio}

\section{Robin Glinatsis}

\section{(2) OpenEdition}

Journals

Édition électronique

URL : http://journals.openedition.org/dictynna/813

DOI : 10.4000/dictynna.813

ISSN : 1765-3142

\section{Référence électronique}

Robin Glinatsis, « Horace et la question de l'imitatio », Dictynna [En ligne], 9 | 2012, mis en ligne le 26 novembre 2012, consulté le 11 septembre 2020. URL : http://journals.openedition.org/dictynna/813 ; DOI : https://doi.org/10.4000/dictynna.813

Ce document a été généré automatiquement le 11 septembre 2020.

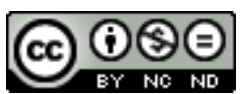

Les contenus des la revue Dictynna sont mis à disposition selon les termes de la Licence Creative Commons Attribution - Pas d'Utilisation Commerciale - Pas de Modification 4.0 International. 


\title{
Horace et la question de l'imitatio
}

\author{
Robin Glinatsis
}

La convocation du ou des modèles auctoriaux est l'une des caractéristiques majeures de l'écriture horatienne. Le poète augustéen éprouve continuellement le besoin de se référer à la figure du primus inuentor, grec dans le cas des Épodes et des Odes, latin dans celui des Satires'. Il s'agit, par l'inscription explicite dans une tradition poétique reconnue, de donner une légitimité forte à ses propres compositions. Il s'agit aussi de définir la place que l'on tient à occuper au sein de cette tradition; faut-il se faire le continuateur fidèle du maître grec (ou latin) ou s'évertuer, quoi qu'il en coûte, à faire preuve d'originalité? En manifestant un intérêt prononcé pour de telles préoccupations, Horace reprend en fait les termes d'un débat séculaire, dont les premières traces apparaissent dans les poèmes de Pindare, de Simonide et de Bacchylide ${ }^{2}$. Au cours de la période hellénistique, la reprise des techniques scripturales, des sujets, des genres donne lieu au développement d'une "rivalité imitative et créative ", non seulement par rapport aux grands modèles, mais aussi entre les poètes alexandrins eux-mêmes ${ }^{3}$. À Rome, le concept d'imitatio accompagne les premières entreprises littéraires et devient rapidement un facteur naturel et, pour ainsi dire, intrinsèque de la littérature latine. Sous l'influence des pratiques poétiques et de la pensée critique alexandrines, les auteurs augustéens s'engagent de manière presque systématique dans une relation d'aemulatio avec leurs prédécesseurs grecs ${ }^{4}$. Toutefois, l'idée de rivalité induite par la notion d'aemulatio ne coïncide que très rarement avec une volonté affichée de surpasser l'exemple grec. Au mieux, les Augustéens aspirent à égaler les performances de leur(s) devancier(s) ; souvent, ils sont amenés, dans le cadre de recusationes ${ }^{5}$, à clamer l'impossibilité de concurrencer le primus inuentor, dont les vers sont conçus comme la représentation la plus aboutie de la forme poétique en question.

Ces considérations traversent l'ensemble du corpus des œuvres d'Horace, selon des modalités et à des degrés variés. Les Épitres proposent un traitement frontal des questions de poétique, alors que les Odes, davantage soumises à la lex operis, travestissent le discours critique qu'elles accueillent. Ainsi les principes éclairant la position d'Horace en matière d'imitatio font-ils l'objet d'une double énonciation: ils 
sont exposés en des termes explicites au sein de l'Épitre I, 19 et de l'Épitre aux Pisons et, à l'aide de procédés spécifiques, prennent une forme actualisée dans certaines odes.

Ces remarques liminaires laissent à penser que les réflexions accordées à l'art des Muses, et en particulier à la relation avec les modèles auctoriaux, se répartissent de manière homogène dans l'œuvre d'Horace ; il paraît alors indispensable, afin de saisir la conception horatienne de l'imitatio dans sa globalité, d'adopter un point de vue transversal. Il nous semble, d'abord, que les considérations tenues sur le sujet à l'intérieur des Épitres ne se limitent pas aux seuls énoncés de l'Épitre I, 19 et de l'Épitre aux Pisons, qui, nous le verrons, tendent à se rejoindre. L'Épittre I, 1, programmatique à plus d'un titre, comporte un passage d'une importance capitale, où le poète exprime avec la plus grande conviction sa volonté d'indépendance. Il s'agit là d'un complément théorique décisif, qui trouve de nombreux points d'ancrage dans la pratique poétique d'Horace.

\section{L'exposé de la conception horatienne dans l'Épître I, 19 et l'Épître aux Pisons}

L'Épitre I, 19, prenant pour champ référentiel les poèmes horatiens eux-mêmes, réserve un traitement particulier au problème de l'imitatio. Archiloque, Alcée et Sappho sont désignés sans détours comme les représentants l'un de la poésie iambique, les autres de la poésie lyrique, et c'est naturellement par rapport à eux que le poète augustéen choisit de se positionner, éclairant par là même la composition de ses Épodes et de ses Odes:

Libera per uacuum posui uestigia princeps, non aliena meo pressi pede. Qui sibi fidet, dux reget examen. Parios ego primus iambos ostendi Latio, numerosque animosque secutus Archilochi, non res et agentia uerba Lycamben. Ac ne me foliis ideo breuioribus ornes, quod timui mutare modos et carminis artem : temperat Archilochi Musam pede mascula Sappho, temperat Alcaeus, sed rebus et ordine dispar, nec socerum quaerit, quem uersibus oblinat atris, nec sponsae laqueum famoso carmine nectit. Hunc ego, non alio dictum prius ore, Latinus uolgaui fidicen ; iuuat inmemorata ferentem ingenuis oculisque legi manibusque teneri.

"J'ai, le premier, porté de libres pas à travers un domaine inexploré ; je n'ai pas foulé de mon pied les traces laissées par un autre. Qui aura confiance en soi conduira l'essaim en chef. Moi, j'ai été le premier à dévoiler au Latium les iambes de Paros, empruntant les mètres et l'esprit d'Archiloque, et non ses sujets ni ses mots qui s'acharnent contre Lycambès. Et ne me couronne pas d'un laurier plus chétif parce que j'ai craint de changer les rythmes et l'art de sa poésie : par son mètre, la virile Sappho tempère la Muse d'Archiloque, Alcée la tempère, mais s'en distingue par les sujets et la disposition; il ne cherche pas un beau-père pour le souiller de la noirceur de ses vers, il ne tresse pas, à l'aide de vers diffamatoires, un lacet pour le cou d'une fiancée. Ce poète, qu'aucune bouche n'avait encore récité, moi, citharède latin, je l'ai le premier révélé. Il me plaît, tandis que j'apporte de la nouveauté, d'être lu par de nobles yeux et d'être tenu par de nobles mains. ${ }^{6}$ 
Si l'on examine attentivement ces quelques vers, on remarque que la figure auctoriale qui représente le point d'origine de la démarche imitative d'Horace est celle d'Archiloque. La référence aux Épodes, dont le poète de Paros constitue le modèle par excellence, s'étend du vers 23 au vers 31, et ce bien que les noms de Sappho et d'Alcée soient cités dès les vers 28 et $29^{7}$. Horace, après une revendication de primauté exprimée en des termes d'inspiration lucrétienne ${ }^{8}$, apporte une restriction décisive à l' imitatio Archilochi à laquelle il a procédé : il ne s'est pas efforcé de restituer les res et les uerba du poète grec, mais en a suivi « les mètres et l'esprit ». Une telle distinction lui permet de signifier son attachement à une tradition qu'il est, à ses yeux, impératif de respecter tout en soulignant l'originalité dont il a fait preuve pour la composition de ses poèmes iambiques.

Les deux versants de l'imitation, positif et négatif, sont glosés dans la suite du passage, notamment grâce à la convocation de Sappho et d'Alcée. Les termes numeros et animos, d'abord, sont repris par modos et carminis artem au vers 27. Les numeri et les modi désignent de façon univoque la structure métrique inhérente à la poésie iambique. Cette dernière se définit avant tout par l'utilisation d'un pied précis, l'iambe, auquel Horace consacrera quelques vers dans l'Épitre aux Pisons ${ }^{9}$. L'auteur augustéen se défend par conséquent du grief qui semble lui avoir été imputé ${ }^{10}$ - une observance trop stricte des modèles grecs - en indiquant qu'il a emprunté aux poèmes archilochiens une caractéristique intrinsèque du genre pratiqué. L'autre élément que le poète iambique ne saurait occulter correspond à ce qu'Horace nomme l'ars carminis. Dans l'économie du passage, la formule ne renvoie pas aux particularités thématiques et / ou formelles de la poésie iambique, mais s'éclaire si, comme nous le préconisions à l'instant, on la rapproche du mot animos employé au vers 24 . Le vocable présente le sens général d'« esprit », de "disposition " ${ }^{11}$, mais dénote également cette colère, cette rage qui anime la poésie archilochienne ${ }^{12}$ et qui s'est rapidement imposée, à son tour, comme une spécificité générique incontournable. Telle est donc la manière dont l'auteur augustéen s'est nourri du matériau textuel offert par le poète de Paros : il s'est appliqué à ne restituer du modèle grec que les éléments indispensables au déploiement du genre. Et de même qu'il s'est appuyé sur le support prosodique transmis par Archiloque, de même Sappho et Alcée, plusieurs fois célébrés dans les Odes en tant que précurseurs du chant lyrique, ont procédé à la reprise du pes Archilochi pour l'élaboration de leurs propres poèmes. L'évocation d'Alcée, en particulier, donne à Horace l'opportunité d'affiner encore la démonstration des principes de l'imitatio. Le syntagme rebus et ordine dispar introduit un nouveau concept, celui d'ordo. E. Pasoli a souligné les divergences interprétatives que suscite l'emploi du mot dans un tel contexte ${ }^{13}$. Certains y ont vu une allusion au système strophique d'Archiloque, qu'Alcée aurait remanié en regroupant les vers par strophes selon des modalités qui lui sont propres. D'autres ont préféré y lire une référence à la disposition de la matière, conformément à l'acception que le terme revêt au cœur de la théorie rhétorique. Nous penchons pour cette seconde lecture, plus adaptée, nous semble-t-il, à l'usage qu'Horace fait du terme dans la perspective adoptée $^{14}$. Le poète lesbien n'a sans doute pas redéfini la structure prosodique caractérisant les compositions archilochiennes, mais s'est plutôt singularisé par un agencement inédit de la matière thématique. Les res qu'Alcée, à l'instar d'Horace luimême, a pris soin de ne pas réitérer font ensuite l'objet d'une glose dans les vers 30 et 31. Il s'agit de ces invectives, évoquées au sein des Épodes ${ }^{15}$, si âpres et diffamatoires que, dit-on, elles poussèrent leurs destinataires au suicide. 
Le lecteur tient donc, dans ces quelques vers, l'essence de la conception horatienne de l'imitatio ${ }^{16}$. L'imitation d'un modèle doit donner le jour à des similitudes d'ordre métrique et stylistique et éviter à tout prix la reprise des sujets que le devancier a mis à l'honneur : tel est le gage de l'originalité en matière de composition poétique. Grâce à cette méthode, Horace sera parvenu à reproduire en vers latins non seulement les rythmes et la vigueur des poèmes archilochiens, mais aussi, en véritable Latinus fidicen, les cadences et l'esprit de la poésie alcaïque. Pour cette question du rapport au modèle, l'Épitre I, 19 apparaît donc comme le pôle théorique de référence et acquiert, au sein de la mosaïque des œuvres d'Horace, une résonance d'autant plus grande qu'elle trouve dans ces dernières des exemples appropriés au sujet traité.

L'intérêt pour le thème de l'imitatio est relayé par l'Épitre aux Pisons, qui, dans l'entrelacs des concepts qu'elle appréhende et des principes qu'elle émet, exhorte notamment l'aîné des fils Pisons, son principal destinataire, à ne pas devenir un fidus interpres:

Publica materies priuati iuris erit, si

non circa uilem patulumque moraberis orbem,

nec uerbo uerbum curabis reddere fidus

interpres, nec desilies imitator in artum,

unde pedem proferre pudor uetet aut operis lex...

«La matière publique deviendra ta propriété privée si tu ne t’attardes pas près du cercle de la banalité et du commun, si tu ne prends pas soin, en fidèle interprète, de rendre le mot par le mot et si tu ne t'engages pas, au cours de ton imitation, dans une voie étroite que la pudeur ou l'économie de l'œuvre t'empêcheront de quitter. » 17

9 Le problème de l'originalité est soulevé en des vers ménageant une transition, plutôt soudaine, du champ du drame vers celui de l'épopée. Mobilisant un lexique emprunté à la sphère légale ${ }^{18}$, le passage constitue un écho manifeste à l'Épître I, 19. En effet, la formule publica materies, privée ici de tout ancrage référentiel, semble particulièrement apte à désigner les cadres métriques et stylistiques offerts par les poésies d'Archiloque et d'Alcée. Les numeri animique du poète de Paros, ses modi et son ars carminis sont des publicae materies qu'Horace, à la suite des chantres lesbiens, a su s'approprier. L'auteur augustéen n'a pas fait œuvre de «traducteur trop fidèle $»^{19}$, ni de simple imitator, mais s'est posé comme un interprète latin à la fois original et respectueux des grands modèles auctoriaux. Il peut, à ce titre, revendiquer une primauté issue d'une bonne connaissance et d'une juste exploitation des canons de la tradition grecque.

Les deux extraits mis en parallèle forment ainsi un socle théorique exposant de manière frontale la façon dont Horace conçoit la relation du poète avec ses modèles, ici à l'aune de la production horatienne elle-même, là dans une perspective plus générale et impersonnelle. Mais cette relation trouve aussi dans certaines odes des espaces textuels propices à sa détermination.

\section{La désignation des modèles dans les Odes : la théorie en acte}

Certains poèmes lyriques proposent une forme actualisée de ce socle théorique. Horace, au moyen de différents procédés, trouve un heureux compromis entre l'expression d'un respect marqué pour les parangons de la poésie lyrique grecque ${ }^{20}$ et la volonté forte et revendiquée de faire jaillir la nouveauté de ses vers. D’ailleurs, la 
lecture des Odes dans cette optique permet de compléter et d'affiner les énoncés théoriques lisibles dans l'Épître I, 19 et l'Épître aux Pisons.

Le Carmen I, 1 place d'emblée les vers horatiens sous l'autorité des poètes de Lesbos et inaugure l'emploi d'un langage métaphorique pour la caractérisation du rapport aux modèles :

Me doctarum hederae praemia frontium

dis miscent superis, me gelidum nemus

Nympharumque leues cum Satyris chori

secernunt populo, si neque tibias

Euterpe cohibet, nec Polyhymnia

Lesboum refugit tendere barbiton.

«Le lierre, qui récompense les doctes fronts, me mêle aux dieux d'en haut, un bois glacé et les chœurs légers des Nymphes accompagnés des Satyres me tiennent éloigné du peuple, si Euterpe ne retient pas sa flûte et si Polhymnie ne se refuse pas à tendre le luth lesbien. $»^{21}$

L'auteur augustéen maniera le «luth lesbien», reprendra l'instrument de ses prédécesseurs et fera résonner, sur les cordes saphiques et alcaïques, son propre chant. $\mathrm{Au}$ sein du Carmen I, 32, il apostrophe ce même barbitos, le priant de «dire un chant latin » sous les auspices d'Alcée :

Poscimus, si quid uacui sub umbra

lusimus tecum, quod et hunc in annum

uiuat et pluris, age dic Latinum,

barbite, carmen,

Lesbio primum modulate ciui,

qui ferox bello tamen inter arma,

siue iactatam religarat udo

litore nauim,

Liberum et Musas Veneremque et illi

semper haerentem puerum canebat

et Lycum nigris oculis nigroque

crine decorum.

«Nous te le demandons, si jamais, l'esprit libre, nous avons joué avec toi sous l'ombre, allons, ô luth, dis un chant qui puisse vivre et cette année et plusieurs autres, dis un chant latin, toi dont les premiers accents furent modulés par le citoyen de Lesbos, ce farouche soldat qui, même au milieu des armes, ou alors qu'il avait attaché au rivage humide son navire agité par les flots, chantait Liber, les Muses, Vénus et l'enfant que l'on trouve toujours à ses côtés, ainsi que le beau Lycus aux yeux et à la chevelure noirs. $»^{22}$

Il apparaît ainsi que la métaphore musicale signifie en des termes adaptés au contexte lyrique la conception horatienne de la relation qu'il convient d'entretenir avec les grandes figures auctoriales de la tradition grecque. En réalité, elle illustre l'affirmation selon laquelle Horace s'est contenté de reproduire le mètre et le style de ses devanciers ; la lyre des Carmina symbolise alors les numeri et les modi évoqués au sein de l'Épître I, 19.

Bien plus, le Carmen I, 32 semble exemplifier l'ensemble de la démonstration dévolue au thème de l'imitatio dans les deux épîtres étudiées. Outre la métaphore de l'instrument, l'ode exhibe une galerie de personnages topiques, strictement attachés, pour certains d'entre eux, au monde romain, alors qu'ils renvoient, dans le cœur de l'énoncé, aux poèmes d'inspiration symposiaque qu'Alcée aimait à composer. En effet, Liber et Vénus, mentionnés au vers 9 , sont des désignations latines de divinités associées à l'univers lyrique depuis l'époque archaïque grecque ${ }^{23}$. Les res lyricae, ces 
sujets et ces motifs récurrents que le dieu du vin et la déesse de l'amour représentent par métonymie, sont donc exposées à l'opération de latinisation de la source alcaïque que le carmen thématise. Dans cette pièce à valeur programmatique, Horace annonce qu'il compte faire des poèmes d'Alcée, dont le corpus peut être considéré comme une «matière publique " que tout poète est libre d'exploiter à sa guise, sa propriété. Il reprendra les grands cadres thématiques de son modèle grec, garants du respect et du maintien de l'ars carminis, mais les confrontera à des éléments proprement latins, en lien avec une conjoncture déterminée, et œuvrera par là même à leur redéfinition. Finalement, les res déployées par l'auteur augustéen différeront de celles d'Alcée : le premier sera, vis-à-vis du second, rebus dispar ${ }^{24}$. La dernière strophe du Carmen I, 32 donne d'ailleurs à voir, toujours par la référence à des figures mythologiques et l'utilisation d'un langage ajusté à l'environnement générique, le résultat auquel doit aboutir le processus d'imitation:

O decus Phoebi et dapibus supremi

grata testudo Iouis, o laborum

dulce lenimen, mihi tcumque† salue rite uocanti.

«Ô honneur de Phébus, lyre chère aux banquets du suprême Jupiter, ô douce consolation de nos tourments, je te salue en t'invoquant selon le rite. $»^{25}$

Le barbitos du début du poème prend la dénomination latine de testudo, comme si Horace avait voulu marquer au niveau lexical l'achèvement de l'entreprise d'imitation, qui, dans son esprit, correspond à une entreprise d'appropriation. De même qu'il affecte les res, le processus touche les uerba ${ }^{26}$; le barbitos grec, celui dont jouait Alcée, est adapté à la spécificité d'une écriture latine et devient, en définitive, une testudo ${ }^{27}$.

Les vues horatiennes en matière d'imitatio trouvent par conséquent des points de résonance explicites au sein du recueil des Carmina, mais s'y expriment aussi de façon plus détournée. Le poète augustéen se plaît, au seuil de certains poèmes lyriques, à insérer ce que Giorgio Pasquali nomme un «motto $»^{28}$ : il s'agit d'un procédé, typiquement horatien, consistant dans la reprise liminaire d'un ou de plusieurs vers grecs issus d'un poème célèbre ${ }^{29}$. L'exemple le plus éloquent se trouve sans doute au premier vers du Carmen I, 37, qui se déploie ensuite selon une dynamique propre :

Nunc est bibendum, nunc pede libero

pulsanda tellus, nunc Saliaribus

ornare puluinar deorum

tempus erat dapibus, sodales.

« À présent il faut boire, à présent il faut frapper le sol d'un pied libéré, c'est à présent le moment, camarades, d'apprêter la couche des dieux pour un festin digne des Saliens. ${ }^{30}$

La formule initiale nunc est bibendum se pose comme la traduction libre d'un vers d'Alcée invitant à célébrer la mort du tyran Myrsilos par l'enivrement :

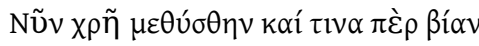

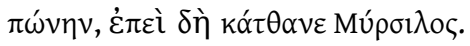

«Maintenant il nous faut l'ivresse; il faut que chacun boive malgré lui, puisque Myrsilos est bien mort ! $»^{31}$

19 Par ce jeu de reprise, la composition se place ainsi sous l'égide d'un modèle grec reconnaissable, dont la convocation, cependant, n'est plus assurée par une mention directe, mais par une sorte d'exercice littéraire. Le carmen procédera bien d'une imitatio Alcaei - d'autant qu'il s'appuie sur l'emploi du mètre alcaïque -, mais cette imitatio sera conforme aux exigences formulées par Horace lui-même en ce domaine. L'adaptation 
au contexte latin du substrat métrique et stylistique laissé par le devancier s'opère avec netteté dès le vers 2 , où allusion est faite aux Saliens, cette confrérie particulièrement active au mois de mars, à l'occasion du Quinquatrus, festival qui, à Rome, marquait l'ouverture de la saison des campagnes militaires ${ }^{32}$. L'insertion du substantif puluinar au vers 3 soutient l'effort d'adaptation amorcé, le terme ayant une résonance proprement latine ${ }^{33}$. S'ensuit un récit aux accents épiques ancré dans l'hic et nunc de la Rome du I ${ }^{\text {er }}$ siècle av. J.-C.; il s'agit en effet de célébrer la victoire d'Actium, symbole du triomphe d'Auguste sur l'Orient, incarné ici par Cléopâtre. Horace a donc bien restitué les numeros animosque des poèmes d'Alcée, mais a veillé à ce que la substance thématique du carmen corresponde à des res spécifiquement latines.

L'expression du rapport au modèle dans les Odes n'emprunte donc pas uniquement la voie de la convocation directe ; elle se fonde aussi sur des allusions voilées, des jeux de translittération qui font appel à la compétence du lecteur, à sa connaissance de la littérature grecque ${ }^{34}$. Finalement, le Carmen I, 37 fournit lui-même un modèle de poème actualisant les principes de l'imitatio édictés par Horace dans l'Épître I, 19. Le recours au «motto » signale l'instauration d'une tension entre le texte sur le point de se déployer et le précédent grec dont il s'inspire. Cette tension, naissant de l'occupation d'un cadre prosodique et stylistique grec par une matière thématique romaine, traduit une forme de déférence envers l'exemplar Graecum en même temps qu'elle assure l'originalité de l'ode latine.

Si l'on considère l'ensemble des Épittres, on s'aperçoit que la question de la relation aux sources n'est pas soulevée qu'au sein de l'Épître I, 19 et de l'Épitre aux Pisons ; l'Épitre I, 1 s'y intéresse aussi, dans une perspective définie.

\section{Complément théorique sur la relation aux modèles dans l'Épître I, 1}

L'épître liminaire du livre I sonne comme un adieu clair et définitif à la poésie, ainsi qu'en témoigne l'assertion suivante :

Nunc itaque et uersus et cetera ludicra pono:

quid uerum atque decens, curo et rogo et omnis in hoc sum.

"C'est pourquoi je délaisse aujourd'hui les vers et toutes les autres bagatelles : je me préoccupe de ce qui est vrai et approprié, je m'interroge sur ce sujet, je suis tout entier absorbé par cette réflexion. $\|^{35}$

Dans ces quelques mots paraissent s'articuler deux moments bien distincts de la carrière littéraire d'Horace. Une tension s'instaure en effet entre deux ensembles syntagmatiques, uersus et cetera ludicra d'un côté, quid uerum atque decens de l'autre, qui apparaissent comme des pôles résolument antithétiques. Le premier a partie liée avec un passé, semble-t-il, révolu, le second avec un avenir amené à se construire sur de nouvelles bases. L'auteur augustéen paraît ainsi renier - ou juger désormais futile et vaine - sa production antérieure ${ }^{36}$, plaçant son écriture sous d'autres enseignes, celles de la philosophie. De fait, les termes uerum et decens ont une résonance philosophique incontestable et caractérisent à eux seuls la nouvelle orientation prise par la pensée horatienne. Le second, en particulier, coïncide de toute évidence avec la notion de $\pi \rho \varepsilon ́ \pi o v^{37}$, élément prégnant de la doctrine stoïcienne soutenue par Panétius ${ }^{38}$; Cicéron en avait proposé une transcription latine, decorum ${ }^{39}$, c'est-à-dire, au sens littéral, « ce qui est approprié », « ce qui convient ». 
Cependant, il ne semble pas que l'attachement à ces concepts, marqués au coin de la réflexion éthique, doive avoir pour corollaire un détachement radical du champ de la poétique, pour lequel Horace avait jusqu'à présent, selon des modalités variées, manifesté un intérêt constant ${ }^{40}$. L'usage cicéronien du terme mentionné, appliqué en priorité au domaine de la rhétorique, ainsi que sa mobilisation récurrente dans l'Épitre aux Pisons, où la préoccupation pour l'art des Muses prédomine, mènent à la conviction que la notion de decens dispose d'une plasticité apte à favoriser son introduction dans les branches de la pensée humaine les plus diverses ${ }^{41}$. «Je me préoccupe de ce qui est vrai et approprié, je m'interroge sur ce sujet, je suis tout entier absorbé par cette réflexion ", dit Horace, sans déterminer pour autant l'ancrage précis de sa quête, sans lever l'ambiguïté inhérente à la largeur référentielle des termes uerum et decens. On peut alors considérer que l'auteur augustéen, malgré la répudiation de ses œuvres passées, projette ici d'examiner et de définir le «vrai » et l'« approprié » en matière de poésie, en même temps qu'il doit sonder, dans une démarche dominée par l'introspection, la sphère de la morale. Considérant l'ensemble du livre I, John Moles souligne en ces termes toute l'ambiguïté du statut et de la portée des Épitres, au confluent de la poésie et de la philosophie: «The Epistles both are poetry and are not poetry, but philosophy; they both are and are not a radical departure, whether in relation to Horace's earlier poetry in general or in relation to his earlier philosophical poetry; and philosophy itself is something that both can and cannot be dissociated from both texts and life ${ }^{42}$.»

Dans ces conditions, la prétention à l'indépendance, que le poète clame au sein des hexamètres suivants, possède une double applicabilité :

Ac ne forte roges, quo me duce, quo lare tuter :

nullius addictus iurare in uerba magistri,

quo me cumque rapit tempestas, deferor hospes.

Nunc agilis fio et mersor ciuilibus undis,

uirtutis uerae custos rigidusque satelles ;

nunc in Aristippi furtim praecepta relabor

et mihi res, non me rebus subiungere conor.

«Et ne va pas me demander sous quel chef, auprès de quel lare je trouve refuge : je

ne me suis pas engagé à jurer sur les paroles d'un maître, mais suis emporté, hôte de passage, partout où m'entraine la tempête. Tantôt je me fais homme d'action et me plonge dans les ondes publiques, gardien et défenseur inflexible de la vraie vertu ; tantôt j'en reviens insensiblement aux préceptes d'Aristippe et m'efforce de dominer les choses sans être dominé par elles. ${ }^{43}$

Horace n'est pas membre d'une domus philosophique identifiable, il n'est rivé de façon entière et définitive à aucun dogme qu'il suivrait scrupuleusement, à l'exclusion de tout autre; c'est un "hôte " prompt à assimiler toute substance spirituelle adaptée à sa propre conception du monde et de la vie ${ }^{44}$. Il a tout loisir de puiser, dans chacun des grands systèmes doctrinaux que sa formation athénienne lui a rendus familiers ${ }^{45}$, les elementa ${ }^{46}$ qui l'aideront à élaborer son propre mode de pensée. Transposé sur le plan de l'écriture poétique, le principe signifie la prise de liberté du poète vis-à-vis des grandes figures auctoriales de la tradition poétique grecque. Suivre la doctrine d'un maître unique reviendrait, dans le domaine de la création littéraire, à traduire avec la plus grande fidélité les œuvres d'un illustre devancier ${ }^{47}$. Horace préfère alimenter sa pensée par des préceptes isolés plutôt que de la circonscrire dans les limites étroites d'un système établi ; de la même manière, il préfère nourrir sa pratique poétique par des 
jeux de référence, par la mise en place d'un art allusif plutôt que de l'enfermer dans une rigoureuse entreprise d'imitation servile.

Ce passage de l'Épitre I, 1 s'érige donc en pôle théorique fort et permet au poète augustéen d'affiner sa conception de l'imitatio. Tâchons à présent d'éprouver le rayonnement de ces quelques vers à l'intérieur du corpus des œuvres d'Horace en nous penchant sur quelques poèmes où se manifeste de façon plus ou moins évidente la volonté de l'auteur de s'affranchir d'un ou de plusieurs modèles donnés.

\section{Lisibilité du précepte dans la pratique poétique d'Horace : une constante volonté d'affranchissement}

Le poème qui, au sein du recueil des Odes, propose l'image la plus nette du souci horatien d'indépendance tel qu'il s'exprime dans l'Épitre I, 1 est le Carmen IV, 2. Face au spectre majestueux et inégalable de Pindare, Horace s'y compare à une abeille besogneuse $^{48}$ :

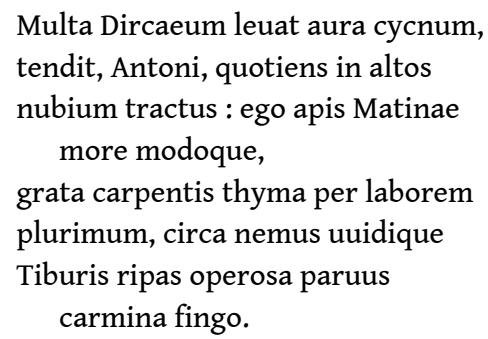

«Un souffle puissant élève le cygne dircéen, Antonius, chaque fois qu'il est attiré vers les hautes nuées; moi, à la manière de l'abeille du Matinus, qui butine l'agréable thym en un effort soutenu, je façonne humblement, dans les bois et sur les rives de l'humide Tibur, des vers issus de mon labeur. ${ }^{49}$

À la différence du cygne dircéen, dont le vol élevé ne connaît pas d'interruptions, l'abeille du Matinus butine le "thym parfumé ", elle se pose de fleurs en fleurs, sans s'attarder particulièrement sur l'une d'entre elles. On peut considérer que ces fleurs sont celles que les poètes grecs ont léguées aux Latins sous la forme de poèmes que leur exemplarité a rendus canoniques ${ }^{50}$. Dans ces conditions, l'abeille et son vol peuvent être assimilés à l'écriture horatienne elle-même, qui se nourrit des fleurs poétiques laissées par les Grecs, mais sans accorder une prédilection exclusive à l'une ou à l'autre ; à peine s'est-elle placée sous l'égide d'un modèle qu'elle ressent le besoin de s'en éloigner, soit par l'attachement à un autre modèle, soit par un traitement personnel du motif ou du sujet repris ${ }^{51}$.

Prenons, pour étayer la démonstration, un exemple précis. Dans l'Épître II, 2, Horace dédie une vingtaine d'hexamètres à la présentation du legitimum poema, du " poème conforme aux règles». L'exposé se fonde en particulier sur une description des différentes opérations liées à la phase de l'electio uerborum :

audebit, quaecumque parum splendoris habebunt

et sine pondere erunt et honore indigna ferentur,

uerba mouere loco (...) ;

obscurata diu populo bonus eruet atque

proferet in lucem speciosa uocabula rerum (...);

adsciscet noua, quae genitor produxerit usus.

Vehemens et liquidus puroque simillimus amni

fundet opes Latiumque beabit diuite lingua. 
«[Celui qui désirera composer un poème conforme aux règles] osera exclure tous les mots qui auront trop peu d'éclat, manqueront de force et seront jugés indignes de tout honneur (...) ; il aura le bon goût d'exhumer et de porter à la lumière des termes expressifs, longtemps ignorés du public (...) ; il en adoptera de nouveaux, que l'usage aura engendrés et produits. Véhément, limpide, semblable à un fleuve pur, il répandra ses richesses et gratifiera le Latium de sa langue opulente. ${ }^{52}$

Avant de déplacer le point focal du discours de l'electio uerborum à la compositio uerborum ${ }^{53}$, l'auteur augustéen introduit une image de facture callimachéenne, celle du fleuve puissant. Sous la plume du poète de Cyrène, cette image exprime un jugement négatif sur les compositions de longue haleine, qui, tel le cours d'eau torrentueux, charrient dans leurs eaux des impuretés ${ }^{54}$. Dans les Satires, Horace l'avait convoquée en maintenant la connotation dépréciative que Callimaque lui avait prêtée. Il s'agissait de stigmatiser le style pléthorique et laborieux de Lucilius $^{55}$, bien éloigné de l'idéal callimachéen incarné par les quelques gouttes de la source sacrée ${ }^{56}$. À la fin du passage mis en exergue, la métaphore est sujette à une inversion connotative, puisque le fleuve, qualifié de " pur $\aleph^{57}$, est pris en bonne part pour désigner symboliquement la langue riche et expressive que le poète aura à sa disposition après avoir éliminé certains mots, en avoir remis d'autres, tombés en désuétude, au goût du jour et en avoir admis d'autres encore, jusque-là confinés à un usage courant, dans le champ de la création littéraire. L'image callimachéenne n'est donc pas reprise de manière univoque ${ }^{58}$, et les modalités de cette reprise trahissent l'inclination horatienne à s'affranchir du joug des modèles en exploitant de nouvelles perspectives, en redéfinissant certains lieux communs.

Un autre exemple de traitement libre et original d'un motif topique se lit dans l' Épode X. La pièce aborde d'un point de vue tout à fait singulier le thème du

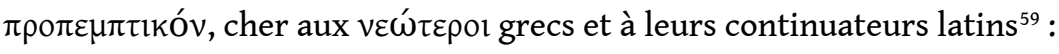

Mala soluta nauis exit alite

ferens olentem Meuium.

Vt horridis utrumque uerberes latus, Auster, memento fluctibus ;

niger rudentis Eurus inuerso mari fractosque remos differat ;

insurgat Aquilo, quantus altis montibus

frangit trementis ilices ;

nec sidus atra nocte amicum appareat, qua tristis Orion cadit (...).

0 , quantus instat nauitis sudor tuis tibique pallor luteus,

et illa non uirilis heiulatio preces et auersum ad Iouem,

Ionius udo cum remugiens sinus Noto carinam ruperit.

«Le navire portant le nauséabond Mévius a levé l'ancre et part sous de funestes auspices. Pense bien, Auster, à en battre les deux flancs en soulevant les flots; que le sombre Eurus tourmente la mer et disperse les câbles et les rames brisées; qu'Aquilon se lève, aussi puissant que sur les cimes des monts, où il brise les chênes tremblants ; qu'aucun astre favorable n'apparaisse dans la nuit noire, là où tombe le sinistre Orion (...). Oh! quelle sueur inondera tes matelots, quelle jaune pâleur s'emparera de ton visage, et ces lamentations dignes d'une femme et ces prières à Jupiter qui te sera hostile, lorsque les flots ioniens, rugissant, auront fracassé la carène avec l'aide de l'humide Notus. $»^{60}$ 

connait l'image callimachéenne du fleuve dans l'exemple précédemment développé. Traditionnellement, il correspond à un poème d'adieu prenant volontiers les accents d'une prière adressée aux divinités marines et / ou aux vents censés favoriser la navigation ${ }^{61}$. Détourné de cette vocation initiale, il sert ici une sorte de deuotio prononcée contre ce Mévius qui ne nous est pas autrement connu, si ce n'est qu'il est mentionné par Virgile dans un vers des Bucoliques ${ }^{62}$. Les éléments topiques de la scène, telles les références aux vents ou aux étoiles, sont bien présents, mais, loin d'être priés de faciliter la course du navire à travers les mers, ils sont appelés les uns à se déchaîner, les autres à rester dissimulées ; le poète leur demande d'œuvrer de conserve à la perte du vaisseau, au lieu de les inviter à en préserver l'intégrité au cours du voyage. On peut légitimement parler, avec Francis Cairns ${ }^{63}$, de $\pi \rho \circ \varepsilon \varepsilon \mu \tau \tau$ ióv inversé. En adaptant ce motif à l'environnement générique de la poésie iambique, Horace manifeste donc, d'une certaine manière, un attachement à l'écriture néotérique en même temps qu'il ménage, par rapport à elle, une forme d'écart.

À l'intérieur du corpus horatien, les exemples de cette sorte sont légion, et la conception horatienne de l'imitatio telle qu'elle se trouve énoncée dans l'Épitre I, 1 ne cesse ainsi de s'actualiser. Le poète augustéen est un " hôte » de passage qui, à l'instar de l'abeille butinant les fleurs, veille à ne pas séjourner trop longtemps dans la même domus poetica. Cette démarche se traduit volontiers par une utilisation originale de motifs relevant d'une tradition identifiable, mais peut aussi se faire jour dans des effets de croisement engageant le procédé de la contaminatio.

La contaminatio, rappelle P. Galand-Hallyn, consiste dans « l'utilisation simultanée de plusieurs modèles $^{64} »$. De fait, un grand nombre de compositions horatiennes se caractérise par des allusions conjointes à des sources distinctes. Les grandes figures auctoriales grecques, convoquées de manière plus ou moins explicite, sont ainsi amenées à cohabiter à l'intérieur des mêmes poèmes. Il suffit, pour le vérifier, de reprendre le Carmen I, 37, que nous évoquions plus haut pour démontrer l'application au corpus lyrique des principes de l'imitatio formulés dans l'Épitre I, 19 et l'Épître aux Pisons. Les considérations émises au sein de l'Épître I, 1 y trouvent à leur tour un solide point d'ancrage. L'ode, nous l'avons observé, s'ouvre sur un «motto » qui augure son positionnement sous l'égide d'Alcée. Pourtant, des références à d'autres modèles ne tardent pas à s'insérer dans le cours du poème, en particulier au moment où est décrit l'assaut de la flotte d'Octave contre les navires égyptiens en déroute :

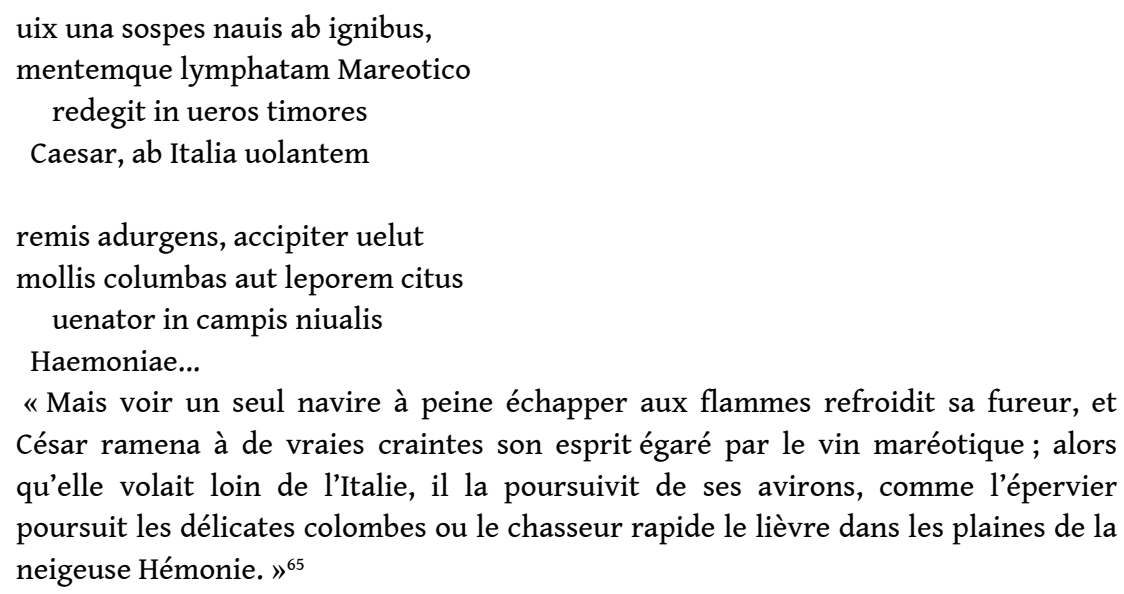


futur Princeps est d'abord comparé à un "épervier» lancé à la poursuite de Cléopâtre, elle-même assimilée à une " timide colombe ». La double comparaison a une origine homérique, puisqu'elle est employée dans l'Iliade pour désigner Achille et Hector lors du combat qui les oppose. Le fils de Pélée, de ses pieds agiles, fond sur son adversaire apeuré, à l'instar du milan poursuivant la craintive palombe ${ }^{66}$. La seconde comparaison fait d'Octave un chasseur traquant la reine d'Égypte, qui, de son côté, prend les traits du lièvre. Le passage ne ménage plus, alors, un écho à l'épopée homérique, mais apparaît comme un souvenir de l'Épigramme XXXI de Callimaque, où, dans un contexte érotique, le poète s'identifie à un chasseur qui «suit les lièvres à la trace »; de même que le second trouve son plaisir dans la traque et ne prend pas la peine de ramasser le gibier tué, de même le premier poursuit de son amour les cœurs qui s'y dérobent et ignore ceux qui sont prêts à lui répondre. Sensible à ce passage inattendu d'une image homérique à une image callimachéenne, Michèle Lowrie y voit la marque d'un raffinement stylistique certain, l'ode s'appliquant ici à " épiciser » le cadre lyrique investi ${ }^{67}$. L'autorité d'Alcée, que le distique liminaire semblait vouloir imposer, se trouve donc subvertie par cette double allusion à Homère et à Callimaque et illustre parfaitement la propension horatienne à la diversification des sources.

En définitive, la poétique d'Horace, qu'elle s'exprime de manière frontale ou qu'elle se reflète dans la pratique littéraire du poète, s'appuie sur une relation au modèle soustendue par l'idée de pluralité. Si quelques-uns, tels Alcée et Pindare dans les Carmina, jouissent d'une relative préséance, personne ne détient le monopole de l'exemplarité. Il apparaît donc que l'Épître I, 1, dans laquelle est énoncé le principe du «butinage » philosophique, apporte un complément théorique de prime importance à la conception horatienne de l'imitatio telle qu'elle se trouve exposée au sein de l'Épitre aux Pisons et surtout de l'Épitre I, 19. L'originalité en matière de création poétique doit naître de la capacité à adapter un cadre métrique et stylistique traditionnel à un contexte linguistique et culturel particulier, mais aussi de l'aptitude à mobiliser conjointement les sources, à les faire coopérer, voire à les fusionner.

Il est édifiant de constater, par l'intermédiaire d'une lecture de type herméneutique, à quel point les pôles théoriques dévolus à la question de l'imitatio se démultiplient à l'intérieur du corpus des œuvres d'Horace. Loin d'opérer un traitement exhaustif du sujet, l'Épittre aux Pisons, que la tradition a assimilée à un pur et simple art poétique, est suppléée par d'autres pièces à forte résonance poétologique. Et chaque principe formulé trouve confirmations et illustrations dans l'écriture horatienne elle-même, qui s'applique à actualiser les fragments de théorie insérés ici et là ; de ce point de vue, il semble finalement possible d'avancer que chaque poème, qu'il soit de facture iambique, satirique, lyrique ou qu'il prenne les traits de l'épître, se pose comme un exemplum éclairant avec plus ou moins de transparence la manière dont Horace conçoit le rapport du poète avec ses modèles. 


\section{NOTES}

1. Cf. l'Épode VI, où allusion est faite à Hipponax d'Éphèse et surtout à Archiloque de Paros, et, entre autres, les Carmina I, 32 et IV, 2, au sein desquels affleurent respectivement les figures d'Alcée et de Pindare.

2. Voir J. WASZINK, Fonction et formes principales de l'imitation dans la poésie préovidienne in Compterendus des séances de l'Académie des inscriptions et belles lettres 116, 1972, p. 240-253.

3. Voir J. GAILLARD, Imitatio, aemulatio : Horace et l'« imitation » in ACD 29, 1993, p. 33-38, à qui est empruntée la formule "rivalité imitative et créative "; c'est en ces termes qu'il définit la notion d'aemulatio. A. Thill (Alter ab illo. Recherches sur l'imitation dans la poésie personnelle à l'époque augustéenne, Paris, 1979, p. 11) commente le phénomène de la façon suivante : «L'imitation littéraire à Rome est inséparable de l'hellénisation. Elle constitue, du point de vue de l'histoire des idées et de l'art, la forme d'appropriation la plus importante par les Romains des biens culturels grecs ; elle est d'abord une condition d'existence qui devient ensuite une tradition. » On se référera également au volume collectif édité par D. WEST et T. WOODMAN, Creative imitation and Latin literature (Cambridge, University Press, 1979).

4. Voir A. LA PENNA, Orazio e l'ideologia del principato, Turin, 1963, p. 180, qui indique que les termes imitatio et aemulatio ne sont soumis à aucune fixation théorique véritable et que, faute de délimitation conceptuelle, ils font souvent l'objet de confusions. «Mais, de fait, précise-t-il, les deux positions, à partir de l'époque augustéenne, existent ".

5. F. KLEIN (La leuitas dans l'œuvre ovidienne. Étude d'une catégorie poétique dans le système littéraire de la Rome augustéenne, Thèse de doctorat soutenue à l'Université de Lille III, 2008, p. 84 sqq.) décrit avec précision l'évolution du motif de la recusatio de Callimaque aux Augustéens. Elle s'attache notamment à montrer que le rejet exprimé dans la recusatio callimachéenne est plutôt d'ordre stylistique, alors que les Romains ont attiré le procédé vers une opposition de type générique.

6. Epist. I, 19, 21-34 (nous proposons nos propres traductions des textes d'Horace que nous citons). Le vers 28 , particulièrement difficile du point de vue de l'analyse grammaticale et, partant, du sens, a fait l'objet d'interprétations diverses; à ce sujet, voir C. W. MCLEOD, The poet, the critic and the moralist: Horace, Epistles 1.19 in CQ 27, 1977, p. 368 ou encore l'article d'A. CUCCHIARELLI, Hor. epist. 1, 19, 28: pede mascula Sappho in Hermes 127, 1999, p. 328-344.

7. Au sujet de la préséance d'Archiloque dans ces vers, voir A. J. Woodman, Horace, Epistle I, 19,

23-40 in $M H$ 40, 1983, p. 76-77.

8. Cf. LUCR. I, 921-927.

9. Cf. Ars, $251 s q q$.

10. À propos du soubassement polémique de l'Épitre I, 19, voir E. CoURBAUD, Horace, sa vie et sa pensée à l'époque des Épîtres, Paris, 1914, p. 318.

11. Voir R. MAYER, Horace. Epistles, Book I, Cambridge, 1994, p. 263.

12. Cf. Ars, 79.

13. E. PASOLI, Le epistole letterarie di Orazio, Bologne, 1964, p. 56-57.

14. L'Épître aux Pisons ménage d'ailleurs une proximité textuelle entre les termes res et ordo, tous deux engagés dans un développement dominé par les concepts rhétoriques d'inuentio et de dispositio (cf. Ars, 40-44).

15. Cf. Epod. VI, 11-16.

16. Nous adhérons pleinement au jugement de C. W. MCLEOD (The poet [n. 6], p. 375-376) selon lequel l'expérience littéraire d'Horace a, dans cet extrait, valeur d'exemple.

17. Ars, 131-135.

18. Voir C. O. BRINK, Horace on Poetry, 2, The « Ars poetica », Cambridge, 1971, p. 209-210. 
19. La formule fidus interpres fait sans doute allusion à la technique littéraire de l'interpretatio, qui, à Rome, consistait dans la traduction d'une source grecque définie (voir, à ce propos, A. REIFF, Interpretatio, Imitatio, Aemulatio. Begriff und Vorstellung literarischer Abhängigkeit bei den Römern, Bonn, 1959, p. 111 sqq.).

20. D. C. FEENEY (Horace and the Greek lyric poets in N. RUDD (ed.), Horace 2000: A Celebration. Essays for the Bimillennium, Londres, 1993, p. 55) rend compte en ces termes de la volonté horatienne de retrouver dans ses vers l'esprit des poésies saphique et alcaïque: "Sappho and Alcaeus in Horace's underworld are not an evocation of a past from which Horace is irredeemably cut off, but an image of a past-in-present which he may join. »

21. Carm. I, 1, 29-34.

22. Carm. I, 32, 1-12. Le poscimus initial est source de désaccord chez les éditeurs modernes. Les commentateurs antiques lui préfèrent le passif poscimur, attesté dans un certain nombre de manuscrits. Les défenseurs de cette lecture justifient leur choix par des références à Pindare (Isthm. VIII, 5 sqq.) et à Ovide (Met. II, 143 sqq., IV, 274 et V, 333), où les verbes ali $\tau \varepsilon \tilde{v}$ et poscere sont employés dans la même perspective. Les partisans de poscimus, quant à eux, reprochent à la leçon poscimur son caractère abrupt et se disent convaincus de la volonté horatienne d'employer ici une formulation caractéristique de la prière ; ils renvoient à Aristophane (Thesm., 1156) et surtout à Pindare (Ol. XII, 1), qui utilise la forme $\lambda$ í $\sigma o \mu \alpha l$ («je prie », «j'adresse une prière ») au seuil de son hymne (voir A. KIEsSLING et R. HeInZE, Q. Horatius Flaccus. Oden und Epoden, Zürich /

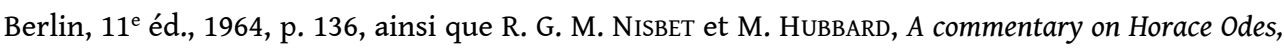
book 1, Oxford, 1970, p. 360). La seconde position nous paraît plus recevable et pertinente.

23. Au sujet du caractère proprement latin du nom de Liber, voir en particulier A. BRUHL, Liber pater. Origine et expansion du culte dionysiaque à Rome et dans le monde romain, Paris, 1953, p. 13 sqq.

24. Cf. Epist. I, 19, 29.

25. Carm. I, 32, 13-16.

26. Cf. Epist. I, 19, 25.

27. A. ERnout et A. Meillet (Dictionnaire étymologique de la langue latine, $4^{\mathrm{e}}$ éd., Paris, 1959, p. 689) précisent que le mot provient du terme latin testa.

28. A. THILl (Alter ab illo [n. 3], p. 155) indique que le terme apparaît dans cette acception au

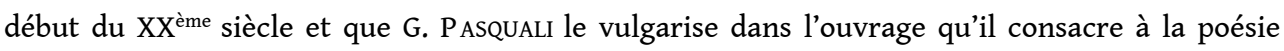
lyrique d'Horace (voir note suivante).

29. Voir G. PASQUALI, Orazio lirico, Florence, 1920, p. 44 sqq. Sur ce point, on consultera aussi et surtout l'ouvrage d'A. CAVARZERE, Sul limitare : Il 'motto' e la poesia di Orazio, Bologne, 1996.

30. Carm. I, 37, 1-4.

31. Fragment 55 dans l'édition de T. ReINACH et A. PuECH parue aux Belles Lettres en 1937 ; traduction de T. REINACH. Tout en relevant la proximité des premiers mots de l'ode horatienne avec ceux du poème d'Alcée, A. FELDHERR ("Dionysiac Poetics" and the memory of civil war in Horace's Cleopatra Ode in B. BREED, C. DAMON, A. ROSSI (éd.), Citizens of discord: Rome and its civil wars,Oxford, 2010, p. 224) note que l'auteur latin s'écarte de son modèle grec dans l'évocation de la mort du tyran. Chez Alcée, la disparition de Myrsilos est clamée dès le deuxième vers et doit, selon le vœu du poète, faire l'objet d'une célébration festive ; chez Horace, Cléopâtre reste en vie jusqu'à la fin du poème, même si son suicide est envisagé dans les trois dernières strophes.

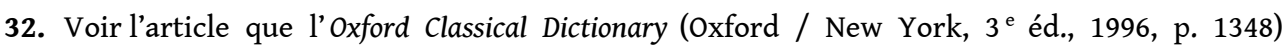
consacre à ce sujet. Il y est notamment précisé que les Saliens sont de naissance patricienne, qu'ils sont liés à Mars et que le faste de leurs repas est proverbial.

33. Voir R. G. M. NISBET et M. HUBBARD, A commentary on Horace Odes, book 1, Oxford, 1970, p. 411. Le puluinar renvoie à un oreiller, un coussin ou plutôt, dans le cadre des lectisternes, au « lit d'apparat " sur lequel on étendait la représentation de la ou des divinités mises à l'honneur (voir aussi A. ERnout et A. M EILlET, Dictionnaire [n. 26], p. 545, qui affirment que malgré 
l'indétermination de son étymologie, le mot est associé à des substantifs (puluillus, puluinulus) et des adjectifs (puluinatus, puluinensis) datant de l'époque impériale).

34. On songe ainsi au concept de « lecteur modèle » tel que le développe U. Eco (Lector in fabula. Le rôle du lecteur ou la coopération interprétative dans les textes narratifs. Traduit de l'italien par M. BOUZAHER, Paris, 1979, p. 61 sqq.).

35. Epist. I, 1, 10-11.

36. Certains commentateurs ont estimé que la formule uersus et cetera ludicra renvoie à l'œuvre lyrique d'Horace stricto sensu (voir E. COURBAUD, Horace, sa vie et sa pensée à l'époque des Épîtres, Paris, 1914, p. 67). Le ton solennel et péremptoire adopté ici par le poète invite plutôt à considérer qu'elle a une portée plus large et qu'elle désigne en réalité toute activité poétique assimilable à un ludus (voir S. J. HARRISON, Horatian self-representation in id. (ed.), The Cambridge companion to Horace, Cambridge / New York, 2007, p. 33).

37. Voir A. D. MorRISON, Didacticism and epistolarity in Horace's Epistles 1 in R. MORELlo et A. D. MoRrison (ed.), Ancient letters. Classical and late antique epistolography, Oxford / New York, 2007, p. 112.

38. Voir M. A. VAN STRAATEN, Panétius : sa vie, ses écrits et sa doctrine avec une édition des fragments, Amsterdam, 1946, p. 160-163.

39. Cic., Orat. 70.

40. Le corpus horatien est émaillé de poèmes poétologiques, c'est-à-dire de pièces qui, en vertu du principe de la réflexivité, émettent des considérations sur leurs propres caractéristiques, thématiques et / ou stylistiques, et, le plus souvent, tiennent un discours de nature théorique sur l'art de la poésie. On pourra ainsi se reporter à l'Épode VI et aux Carmina I, 32 et IV, 2, déjà mentionnés dans la note 1, mais aussi, entre autres compositions, aux Satires I, 4, I, 10 et II, 1 ou encore aux Carmina I, 6, II, 12 et III, 30.

41. Il suffit de consulter l'article que le TLL consacre au mot (V, 1934, col. 135-137) pour réaliser que cette plasticité tient non seulement à l'adaptabilité de son sens premier ("approprié », « convenable ») aux contextes les plus variés, mais aussi à l'existence d'un sens second, qui tend à en faire un terme directement lié au domaine esthétique.

42. J. MOLES, Philosophy and ethics in S. J. HARRISON (ed.), The Cambridge companion to Horace, Cambridge / New York, 2007, p. 176.

43. Epist. I, 1, 13-19.

44. Si l'on y regarde bien, le passage procède d'un enchevêtrement d'allusions à d'illustres principes philosophiques empruntés aux grandes écoles de l'Antiquité. Le vers 14 fait écho à un extrait des Tusculanes dans lequel Cicéron affirme, en des termes proches de ceux qu'emploie Horace, sa propre volonté d'indépendance à l'égard des grands systèmes doctrinaux (cf. Tusc. IV, 7). Le vers 15 rappelle un autre développement cicéronien, cette fois issu des Académiques (cf. Acad. II, 8), où domine aussi l'idée d'une autonomie de la pensée; le lien intertextuel se tisse grâce à la métaphore de la tempête. Le mot hospes lui-même, mobilisé dans un tel contexte,

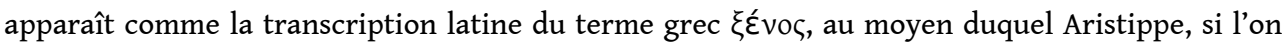
en croit Xénophon, qualifiait sa propre posture philosophique (cf. XEN., Mem. II, 1, 13). Quant au vers 19, il évoque la conviction d'Aristippe, telle que la reproduit Diogène Laërce, selon laquelle il est permis, voire recommandé de jouir des plaisirs de la vie, à condition de ne pas se laisser asservir par eux (cf. DIOG. LAERT. II, 74-75). Au sujet de l'utilisation horatienne de la pensée d'Aristippe, voir A. TRAINA, Orazio e Aristippo. Le Epistole e l'arte di convivere in RFIC 119, 1991, p. 285-305.

45. Voir K. RECKFORD, Pueri ludentes: Some aspects of play and seriousness in Horace's Epistles in TAPhA 132, 2002, p. 5, qui affirme que les Épîtres marquent le retour d'Horace aux préoccupations philosophiques qu'il avait affichées à Athènes, de nombreuses années auparavant.

46. Cf. Epist. I, 1, 27. 
47. Bien plus qu'un poeta, dont A. ThiLl (Alter ab illo [n. 3], p. 12) rappelle le sens ancien de "traducteur " aux côtés de celui de " poète ", Horace est un uates, titre pouvant illustrer à lui seul l'aspiration des Augustéens à adapter les données de la tradition grecque à un contexte spécifiquement latin.

48. Horace mobilise aussi l'image de l'abeille au sein de l'Épitre I, 19; voir J. KATZ, Dux reget examen (Epistle 1.19.23): Horace's Archilochean signature in Materiali e Discussioni per l'analisi dei testi classici 59, 2007, p. 207-213.

49. Carm. IV , 2, 25-32.

50. Au sujet du lien métaphorique entre la fleur et le poème, cf. Carm. I, 26.

51. À propos de l'image de l'abeille dans ce carmen, voir notamment S. J. HARRISON, Horace, Daedalus, Pindar, and Augustus: Odes 4.2 in id. (éd.), Homage to Horace: A bimillenary celebration, Oxford, 1995, p. 114. Cette image est déjà présente chez Lucrèce, qui s'imagine butinant les fleurs de la pensée épicurienne (cf. LucR. III, 11-13).

52. Epist. II, 2, 111-121.

53. Pour ce qui concerne la compositio uerborum, cf. Epist. II, 2, 122-125. Il s'agit là de formules conceptuelles empruntées à la théorie rhétorique (voir C. O. BRINK, Horace on Poetry. Epistles Book II: The Letters to Augustus and Florus, Cambridge, 1982, p. 343).

54. Cf. Hymn. Ap., 108-109.

55. Cf. Sat. I, 4, 9-13. La double métaphore callimachéenne du fleuve et de la source est déjà convoquée dans la Satire I, 1 (v. 54-60).

56. Cf. Hymn. Ap., 110-112.

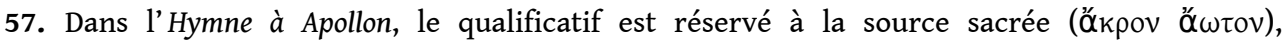
précisément opposée au fleuve limoneux.

58. À propos du phénomène de "politisation » de l'idée callimachéenne à l'œuvre dans ce passage, voir K. FREUDENBURG, Writing to / through Florus: criticism and the addressee in Horace Epistles 2.2 in id. (éd.), Horace: Satires and Epistles, Oxford, 2009, p. 420-421.

59. Voir L. AlfoNSI, Poetae novi: storia di un movimento poetico, Côme, 1945, p. 112.

60. Epod. X, 1-20.

61. L'une des premières convocations du motif se trouve chez Théocrite (cf. Id. VII, 52-62). Il est notamment repris par Callimaque et par Horace lui-même, qui, dans le cadre du Carmen I, 3, en fait un usage conforme à la tradition.

62. Cf. Ecl. III, 90.

63. Voir F. CAIRNS, Generic composition in Greek and Roman poetry, Edinburgh, 1972, p. 130. L'épode prend en fait le contre-pied du Propemptikon Pollionis que Cinna avait écrit pour son ami Pollion. Le poème horatien établit aussi un lien énonciatif entre deux poètes, mais le place sous le signe de l'antagonisme (au sujet des $\pi \rho 0 \pi \varepsilon \mu \pi \tau$ ' $\alpha$ antérieurs à Horace, voir L. C. WATSON, A commentary on Horace's Epodes, Oxford, 2003, p. 342).

64. P. GALAND-HALLYN, Le reflet des fleurs : description et métalangage poétique d'Homère à la Renaissance, Genève, 1994, p. 101

65. Carm. I, 37, 12-20.

66. Cf. Il. XXII, 136-144.

67. Voir M. LowRIE, Horace's narrative Odes, Oxford, 1997, p. 157. Dans les pages suivantes, M. LOWRIE tire grand parti du réseau de comparaisons que ces vers instaurent. 


\section{RÉSUMÉS}

La conception horatienne de l'imitatio se lit dans deux passages respectivement issus de l'épitre I, 19 et de l'Épitre aux Pisons. Horace y soutient que l'originalité en matière de création poétique doit naître de la capacité à adapter un cadre métrique et stylistique traditionnel à un contexte linguistique et culturel particulier. En outre, au seuil de l'Épittre I, 1, il souligne son penchant pour le «butinage » philosophique : il ne se rangera jamais sous la bannière d'un seul maître, mais prélèvera au sein de chaque doctrine les éléments aptes à nourrir sa propre réflexion. Une telle assertion semble également pouvoir trouver un terrain d'application dans le rapport que le poète souhaite entretenir avec les grands modèles auctoriaux de la tradition grecque. Il ne s'agit pas de s'inscrire dans le sillage étroit d'un même devancier, mais de mobiliser les sources conjointement, de les faire coopérer, voire de les fusionner. Quelques exemples puisés au cœur du corpus horatien ont tôt fait de le démontrer.

INDEX

Mots-clés : contaminatio., Horace, imitatio, originalité, théorie en acte, tradition grecque 